Military Technical College Kobry El-Kobbah, Cairo, Egypt

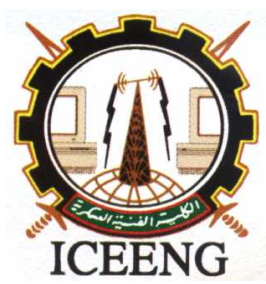

\author{
$6^{\text {th }}$ International Conference \\ on Electrical Engineering \\ ICEENG 2008
}

\title{
DESIGN OF A ROBUST CONTROLLER FOR ANTENNA TRACKING SYSTEM USING RECESSIVE TRAIT CROSSOVER GENETIC ALGORITHM
}

By

A. A. El-Mahallawy *

H.Y.Abd-halim**

A. A. Madkour****

M.I. El-Singaby ***

\section{Abstract:}

This paper presents an investigation into the optimal design of a robust controller for an antenna tracking system. Evolutionary Genetic Algorithm (GA) is used to design controllers for an airport radar antenna pointed towards an air plane to track its movement. This is achieved by minimizing the error between the over all candidate system and the ideal one according to limited specifications. Finally, the merits of the candidate controller for an antenna tracking system is presented and discussed through a set of experiment.

\section{Keywords:}

Antenna Tracking System, Genetic Algorithm, Recessive Trait Crossover Genetic Algorithm, Robust Controller,

** College of Engineering, Alexandria University, Alexandria, Egypt

*** College of Engineering \& Technology, Alexandria, Egypt

**** Egyptian Armed Forces, Military Technical College, Cairo, Egypt 


\section{Introduction:}

Many researchers succeeded to design controllers for an airport radar antenna pointed towards an air plane to track its movement [1-4]. Parameter variations, disturbance and white noise were taken into considerations to check for the robustness of controllers. In this paper a Recessive trait crossover genetic algorithm (RCGA) [5] is used in the design of a low order robust controller for the antenna tracking system.

The rest of the paper is organized as follows. In section 2, the recessive trait crossover genetic algorithm is introduced, while the antenna tracking system has been described in section 3. Simulation results are carried out in section 4 to show the superiority of the modified genetic algorithm. Concluding remarks are found in 5.

\section{Recessive trait crossover genetic algorithm:}

In the nineteenth century, Darwin originated his theory of evolution [6-8]. Darwin suggested that in the universal struggle for life, nature "selects" those individuals who are best suited (fittest) for the struggle, and these individuals in turn reproduce more than those who are less fit, thus changing the composition of the population.

There are three methods of population inheritance, dominant, recessive and sex linked. [9]. The sex-linked properties expressing depend on the person sex. For dominant properties, only one genetic trait is needed for this property to be expressed. However, if a genetic trait is recessive, a person needs to inherit two copies of the gene for the trait to be expressed. Thus, both parents have to be carriers of a recessive trait in order for a child to express that trait. If both parents are carriers, there is a $25 \%$ chance with each child to show the recessive trait and it becomes $100 \%$ if the both have that recessive trait.

Using the concepts taken from the recessive property inheritance a crossover operator has been developed. Here the GA with this operator is called recessive trait crossover GA (RCGA). The RCGA produces children by selecting the common genes between parents, and choosing the remaining genes randomly. The main difference between the traditional crossover GA (TCGA) and RCGA is the way of how the new population is inherited from the previous generations. To use the proposed population inheritance approach through the recessive trait crossover we assume that the complementary of all of the chromosome parts makes its survival fitness, and the length of the chromosomes is fixed.

Let us assume that two parents have the eight genes chromosome as shown in Table 1. It is worth noting that these parents have common genes at (1, 3, 5, and 6). According to Darwin theory, these two parents struggle fitness depending on their common genes, so we can keep these genes without any change when we produce their children to fit the 
same struggle fittest and try to make there children of better fitness by crossover the different genes using the four possible binary combinations randomly. This is the only random operation in our TCGA. The new solutions will be as shown in Table 2.

\begin{tabular}{|c|cccccccc||}
\hline \hline Table 1: An example of & \multicolumn{1}{c||}{ Two parents have } & genes chromosome \\
\hline \hline Gene no. & $\mathbf{1}$ & $\mathbf{2}$ & $\mathbf{3}$ & $\mathbf{4}$ & $\mathbf{5}$ & $\mathbf{6}$ & $\mathbf{7}$ & $\mathbf{8}$ \\
\cline { 2 - 10 } & 0 & 1 & 1 & 0 & 0 & 1 & 1 & 0 \\
Parent 2 & 0 & 0 & 1 & 1 & 0 & 1 & 0 & 1 \\
\hline
\end{tabular}

\section{Table 2: The reproducing in table 1 using RCGA}

\begin{tabular}{|c|cccccccc||}
\hline Gene no. & $\mathbf{1}$ & $\mathbf{2}$ & $\mathbf{3}$ & $\mathbf{4}$ & $\mathbf{5}$ & $\mathbf{6}$ & $\mathbf{7}$ & $\mathbf{8}$ \\
\cline { 2 - 9 } Child 1 & 0 & 1 & 1 & 1 & 0 & 1 & 0 & 0 \\
Child 2 & 0 & 1 & 1 & 0 & 0 & 1 & 1 & 0 \\
Child 3 & 0 & 0 & 1 & 0 & 0 & 1 & 0 & 1 \\
Child 4 & 0 & 0 & 1 & 1 & 0 & 1 & 1 & 1 \\
\hline
\end{tabular}

Referring to recessive trail behavior, the selection of the parents for mating is very important. We have used a selection operation which sorts the old populations according to their fitness and then reproducing the first parent with the second to generate four new populations, as shown in the above example, and so on.

The overall algorithm can be written as:

1. Create a random population of $\mathrm{N}$ individuals

2. Evaluate their fitness.

3. Sort the individuals in the population according to their fitness.

4. Choose the best N/2 individuals as mating pool to generate the new population.

5. Generate four new individuals by reproducing the nearest two parents from the mating pool keeping the common genes and randomly swapping the different genes. This creates a new population of $\mathrm{N}$ individuals.

6. Apply mutation operation with a probability.

7. Repeat steps from 2 to 6 for the best fitness value.

This RCGA offered better convergence, higher accuracy, and it is very sample and easy to use for any numerical optimization problem as a minimum tool [10]. 


\section{The Antenna tracking system description:}

An airport radar antenna is pointed towards an airplane to track its movement [1]. A remote control system, needed to locate the antenna towards the target comprises a ward-Leonard system which is a generator motor connection. The system contains a D.C generator used as a power amplifier of the control signal "u". The D.C generator being rotated by a constant speed from a prime mover, an output voltage $\mathrm{Vg}$ is generated and supplies the armature of an antenna controlled D.C motor. The exciting winding of the motor is fed by a D.C external supply. Movement of the motor axis is coupled to the antenna through a gear box. The connection diagram of the individual plant components is shown in figure (1), together with the nomenclature of each element. Figure (2) shows the block diagram of the antenna tracking system.

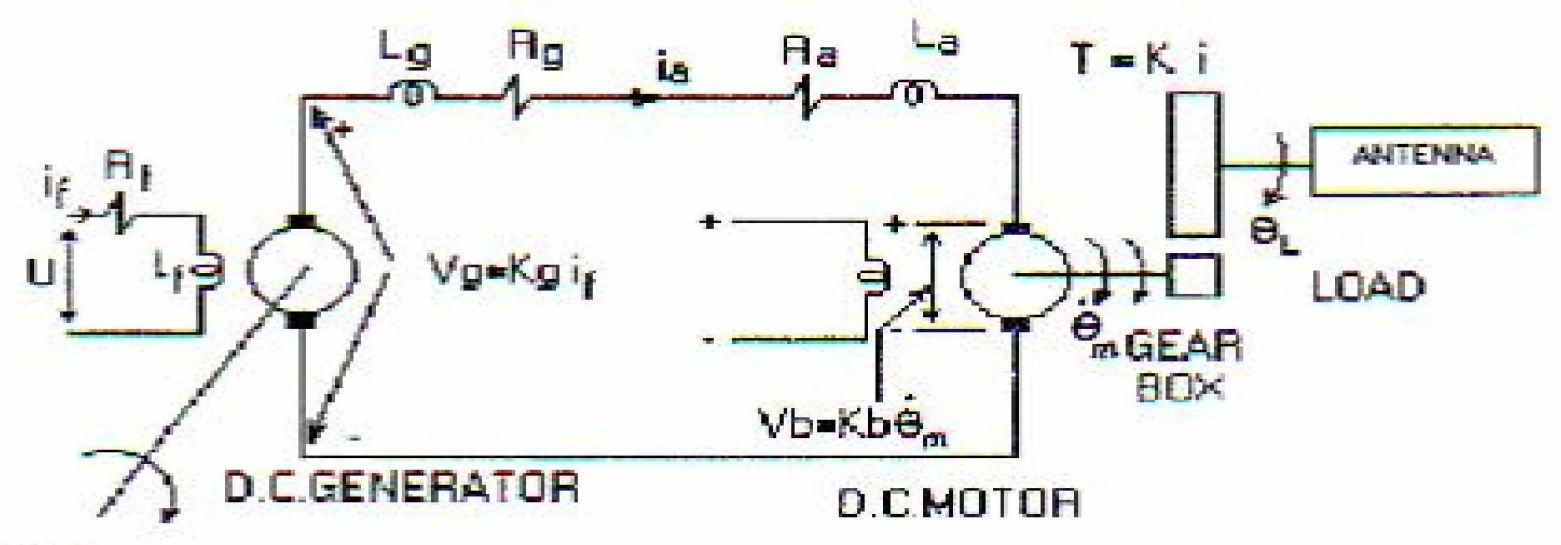

FROM

PRIMOVER

Figure (1): Plant model of the antenna tracking system

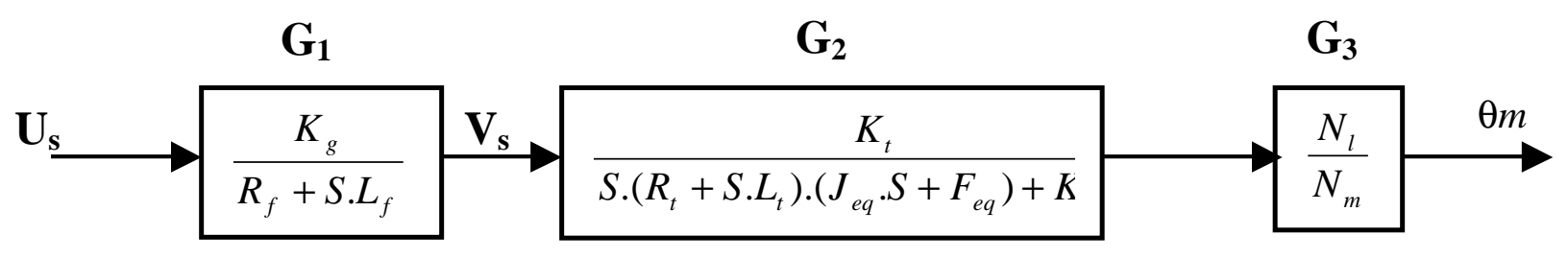

Figure (2): The Block diagram of the antenna tracking system 
It is worth noting that, the transfer function of this system can be obtained from figure (2) as the following:

$$
\begin{aligned}
& G_{1}(s)=\frac{V_{g}(s)}{U(s)}=\frac{K_{g}}{R_{f}+s L_{f}} \\
& G_{2}(s)=\frac{\theta_{m}(s)}{V_{g}(s)}=\frac{K_{t}}{s\left[s J_{\text {meq }}+F_{\text {meq }}\right]\left[R_{t}+s L\right]+K_{t} K_{b} s}
\end{aligned}
$$

The load angle $\theta_{L}$ is related to the motor angle $\theta_{m}$ by:

$$
\frac{\theta_{L}(s)}{\theta_{m}(s)}=\frac{N_{m}}{N_{L}}
$$

where

$R_{t}=R g+R a, L_{t}, L_{g}, L_{a}, L_{b}, K_{b}$ and $K_{t}$ are constant

The torque at the motor end:

$T=K_{t} L_{a}=J_{\text {meg }} s^{2} \theta_{m}(s)+F_{m e g} s \theta_{m}(s)$

\section{Simulation results:}

To demonstrate the effectiveness of the RCGA as compared to the $\mathrm{H} \infty$, The open loop transfer function of the overall antenna tracking system $\mathrm{G}(\mathrm{s})$ can be calculated by combining equations $(1,2,3$ and 4$)$ using the following data system.

$$
\begin{array}{lll}
\frac{N_{m}}{N_{L}}=0.5 & R_{f}=20 \Omega & L_{f}=5 \mathrm{H} \\
R_{g}=9 \Omega & L_{g}=0.06 \mathrm{H} & R_{a}=9 \Omega \\
L_{a}=0.04 \mathrm{H} & J_{L}=1.6 \mathrm{~N} \cdot \mathrm{rad}^{-1} & F_{L}=0.04 \mathrm{~N}_{m} \cdot \mathrm{rad}^{-1} \cdot \mathrm{Sec} \\
K^{t}=1.2 N_{m} / \mathrm{A} & L_{g}=0.06 \mathrm{H} & K_{g}=100 \mathrm{~V} / \mathrm{A}
\end{array}
$$




$$
G(s)=\frac{\theta_{L}(s)}{U(s)}=\frac{300}{s\left(s^{3}+184 s^{2}+760.5 s+162\right)}
$$

It is worth noting that, moments of inertia of the motor, gearbox and transducers are very small and are not included in equation (5).

The saturation characteristic occurs in the generator winding makes the representation of the plant by transfer function (5) incorrect unless the control $U_{t}$ is bounded to its linear range by:

$$
|U(t)| \leq K ; t \geq 0
$$

where $\mathrm{K}$ is a given positive number. Under this condition, the relation between the output $\theta_{L}$ and the control signal is linear, time invariant and the equations $(5,6)$ presents a conditionally linear model. The design of control systems for conditionally linear plants presents severe difficulties to those standard methods of design such as Nyquist, and root-locus methods.

The design problem can be restated as to design a cascade controller $\mathrm{K}(\mathrm{s})$ such that the overall antenna tracking system satisfies the follows constraints :

1. Closed loop stability

2. Settling time

$$
\begin{aligned}
& T_{s}=20 \mathrm{Sec} . \\
& T_{r}=5 \mathrm{Sec} . \\
& T_{p}=6 \mathrm{Sec} . \\
& P_{0}=18 \% \\
& M_{p}=1.2 \\
& G=10 \mathrm{~dB} \\
& \theta=80^{\circ}
\end{aligned}
$$

3. Rise time

4. Peak time

5. Percentage over shoot

6. The controller output

7. Gain margin

8. Phase margin

The same case of study has been done by [E. Aziz et al, 2006] [3].

They obtained a $4^{\text {th }}$ order controller using $\mathrm{H} \infty$ as :

$$
K(s)=\frac{4.468 s^{3}+822.7871 s^{2}+3505.3384 s+1143.1957}{s^{4}+187.6127 s^{3}+142.5281 s^{2}+3514.952 s+3341.8312}
$$


It is observed that 100 generations, 16 bit representation, $10 \%$ mutation rate, and 50 population sizes the RCGA, produced the following $2^{\text {nd }}$ order controller

$$
K(s)=\frac{18.7341 s+4.7642}{s^{2}+4.9119 s+12.7562}
$$

Table 3 shows the summary of the overall antenna tracking system time response in implementing both controller ( $\mathrm{H} \infty$ and RCGA) with respect to the candidate system constraints. Figures 3,4 show the time and frequency response of the closed loop system using $\mathrm{H} \infty$ and RCGA.

\begin{tabular}{|l|c|c|c|}
\hline Value & candidate system & $\mathrm{H} \infty$ & RCGA \\
\hline$T_{p}($ Sec. $)$ & 6 & 6.03407 & 4.64133 \\
\hline$T_{r}($ Sec. $)$ & 5 & 2.4136 & 1.87863 \\
\hline$T_{s}($ Sec. $)$ & 20 & 10.8372 & 6.37261 \\
\hline$P_{0}(\%)$ & $18 \%$ & 17.1291 & 2.8 \\
\hline$G(d B)$ & 10 & 11.5 & 11.5 \\
\hline$\theta(0)$ & 80 & 87.2 & 147 \\
\hline
\end{tabular}
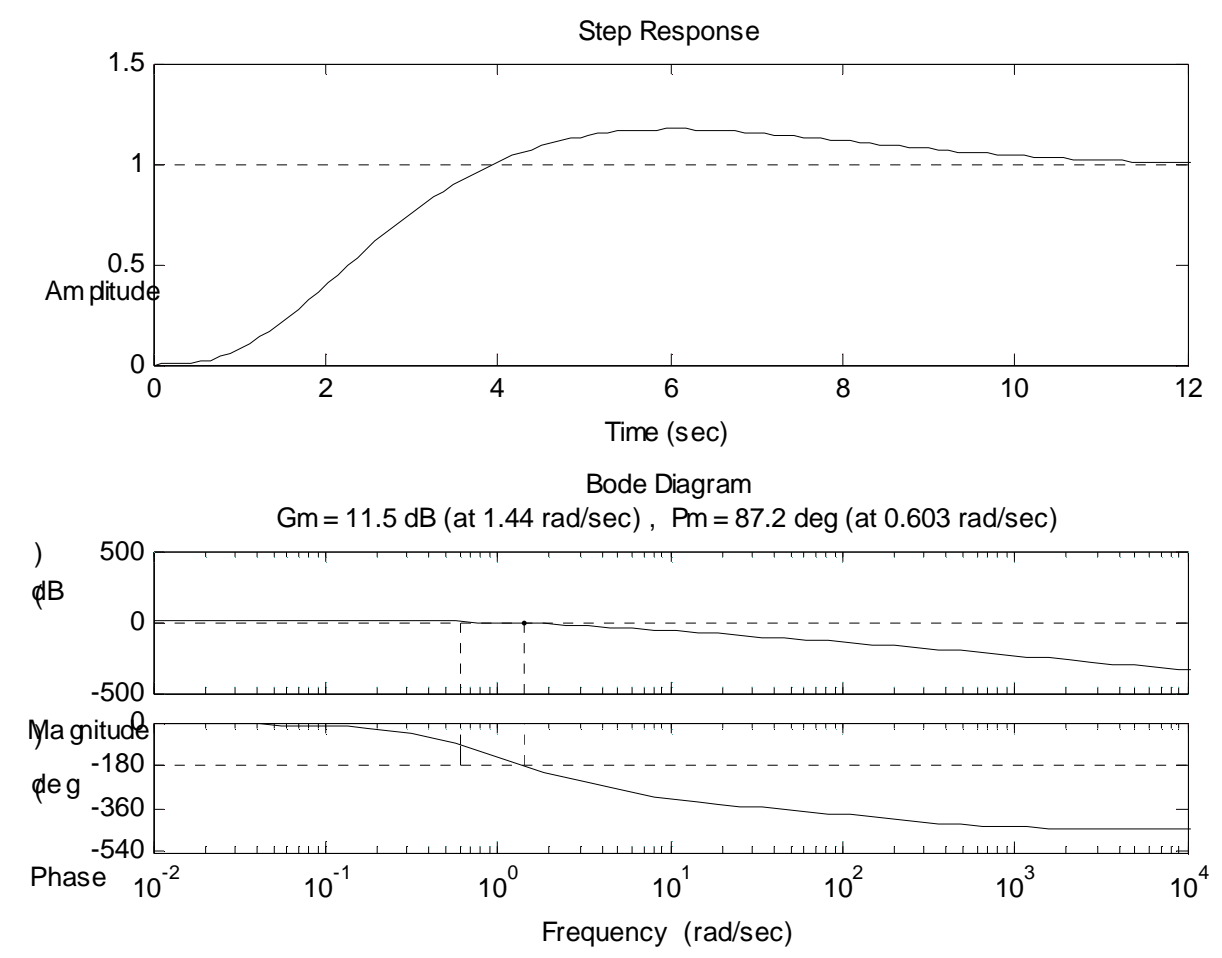

Figure (3): Time and frequency response of the closed loop system using $H \infty$ 


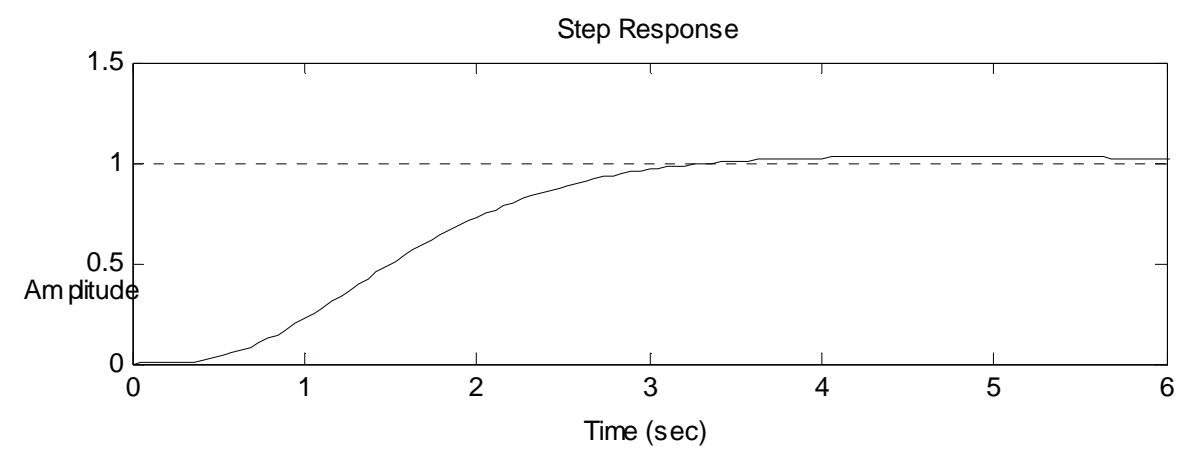

Bode Diagram

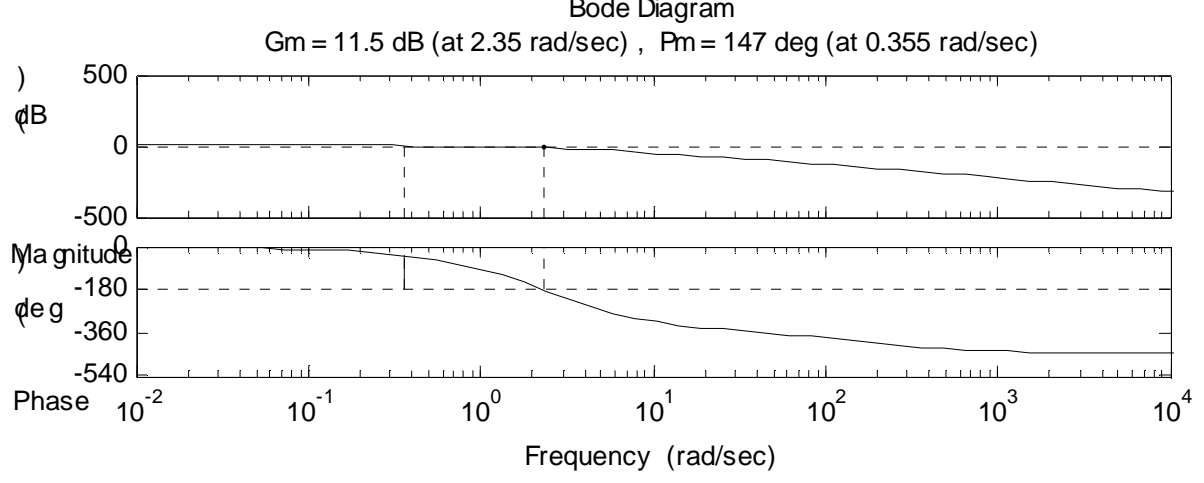

Figure (4): Time and frequency response of the closed loop system using RCGA.

To demonstrate the effectiveness of the candidate controller further in, let's consider $10 \%$ variation in the open loop gain for the two cases.

Table 4 shows the summary of the overall antenna tracking system time response in implementing both controller ( $\mathrm{H} \infty$ and RCGA) with respect to the candidate system constraints. Figures 5,6 show the time and frequency response of the closed loop system using $\mathrm{H} \infty$ and RCGA.

\begin{tabular}{|l|c|c|c|}
\hline Value & candidate system & $\mathrm{H} \infty$ & RCGA \\
\hline \hline$T_{p}($ Sec. $)$ & 6 & 5.55398 & 4.22975 \\
\hline$T_{r}($ Sec. $)$ & 5 & 2.18269 & 3.82976 \\
\hline$T_{s}($ Sec. $)$ & 20 & 9.89776 & 1.59883 \\
\hline$P_{0}(\%)$ & $18 \%$ & 19.4375 & 5.8376 \\
\hline$G(d B)$ & 10 & 10.4 & 10.4 \\
\hline$\theta(0)$ & 80 & 78.9 & 139 \\
\hline
\end{tabular}




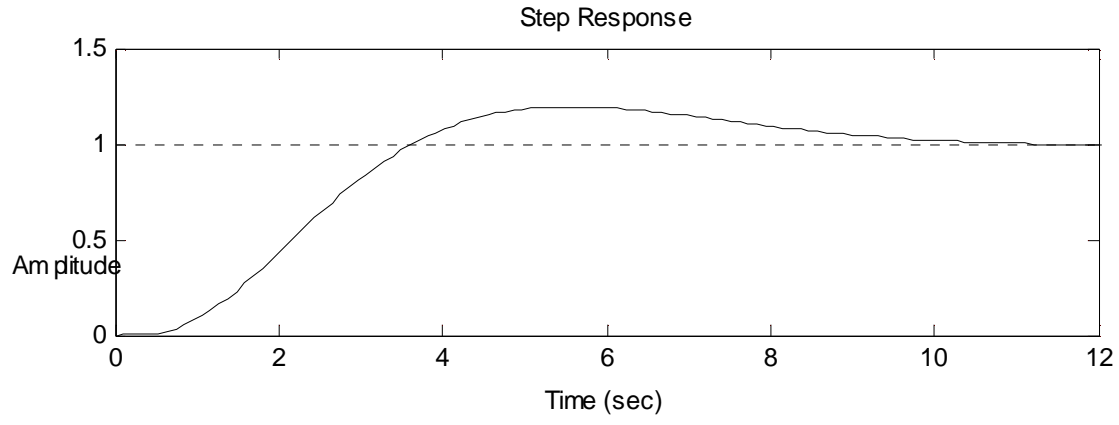

Bode Diagram

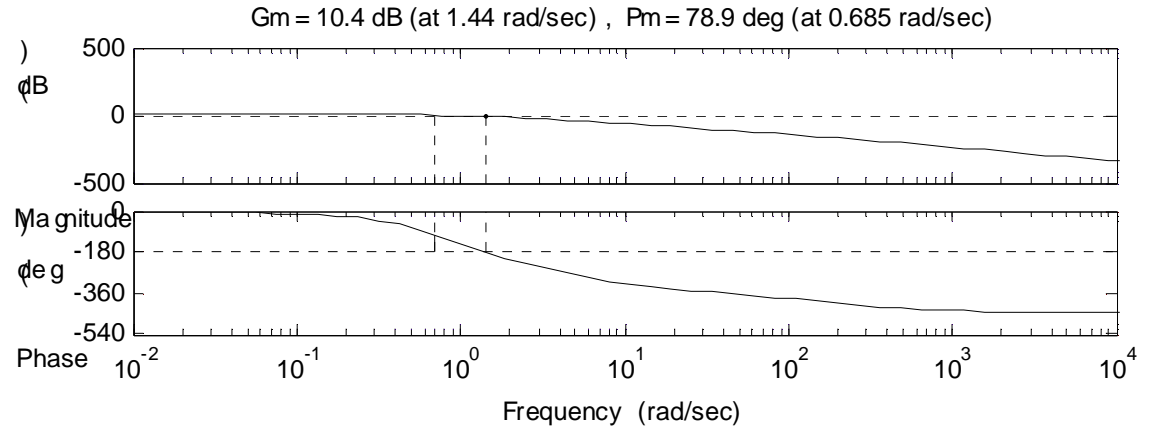

Figure (5): Time and frequency response of the closed loop system using $\mathrm{H} \infty$ with $10 \%$ gain variation

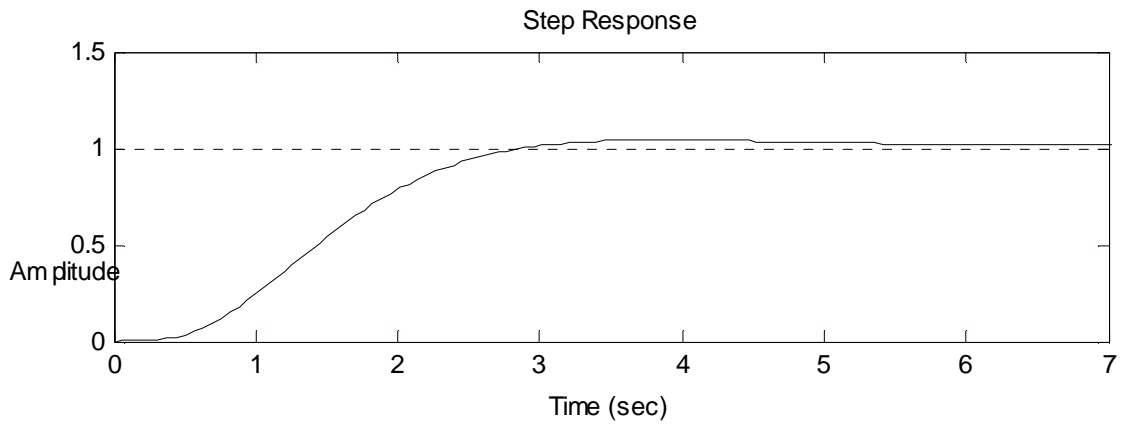

Bode Diagram

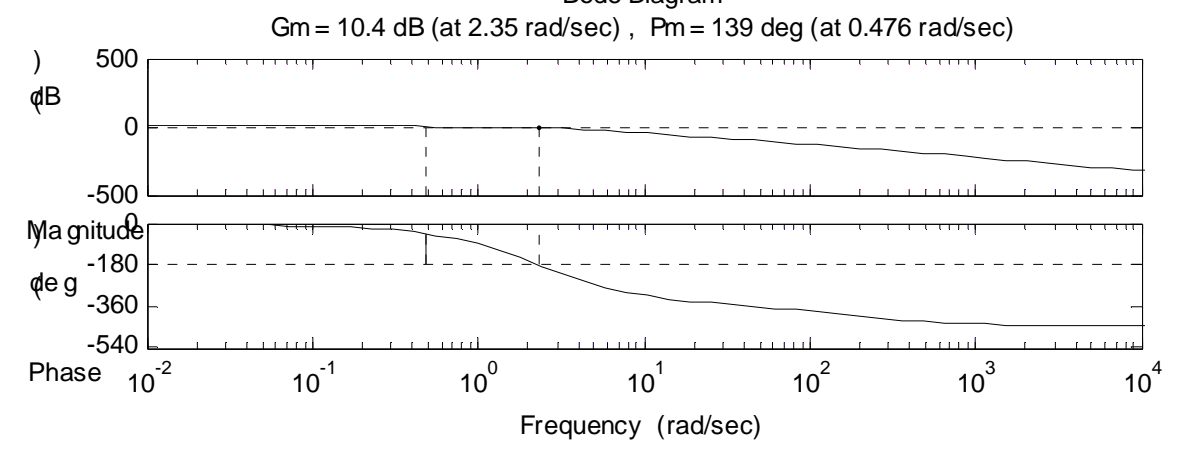

Figure (6): Time and frequency response of the closed loop system using RCGA with10\% gain variation 
To demonstrate the effectiveness of the candidate controller furthermore, let's consider an existence of disturbance of amplitude (-0.001) of reference step up for the two cases. Figures 7,8 show the time and frequency response of the closed loop system using $\mathrm{H} \infty$ and RCGA.

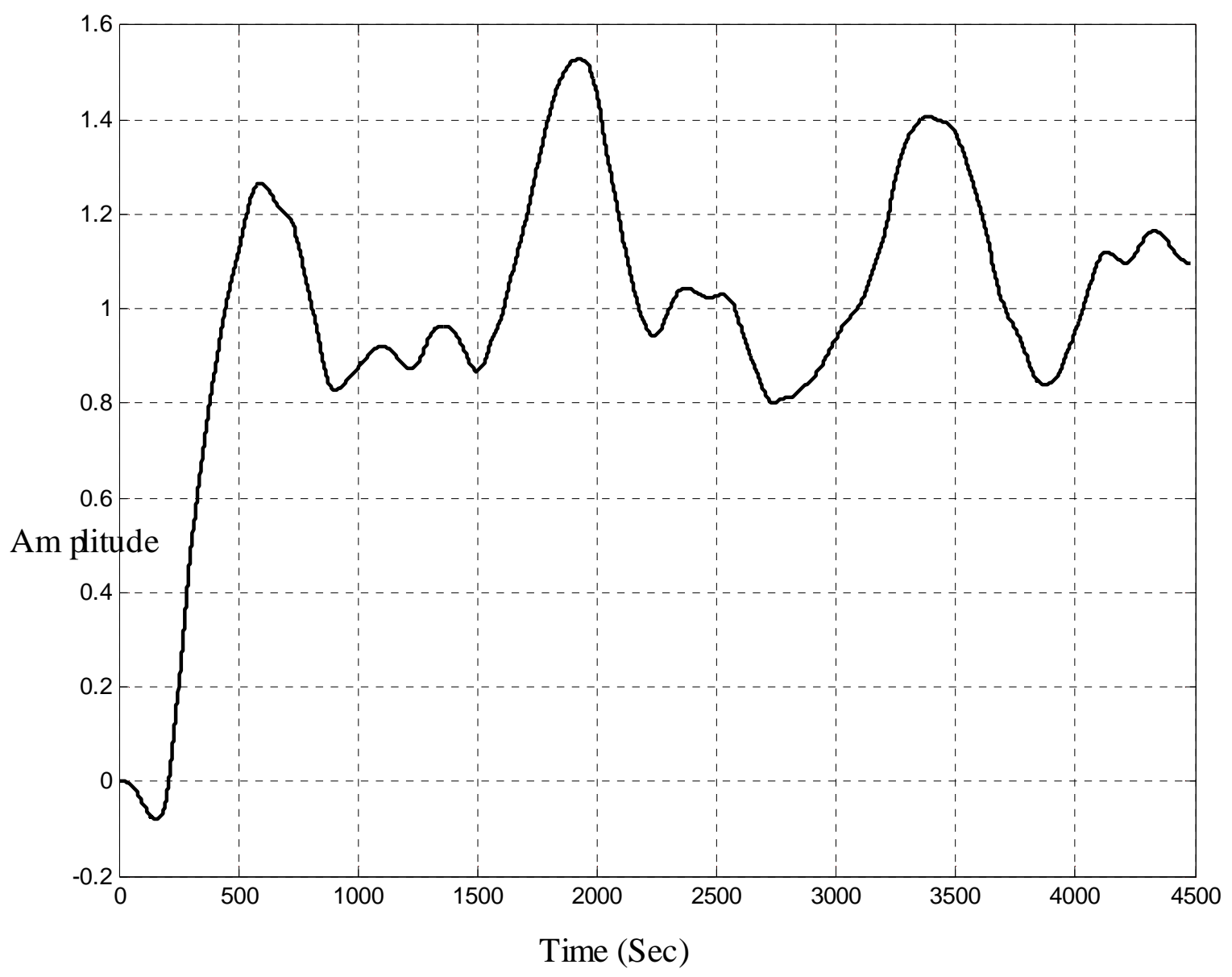

Figure (7): Time response of the closed loop system using $H \propto$ with (-0.001) existence of amplitude disturbance 


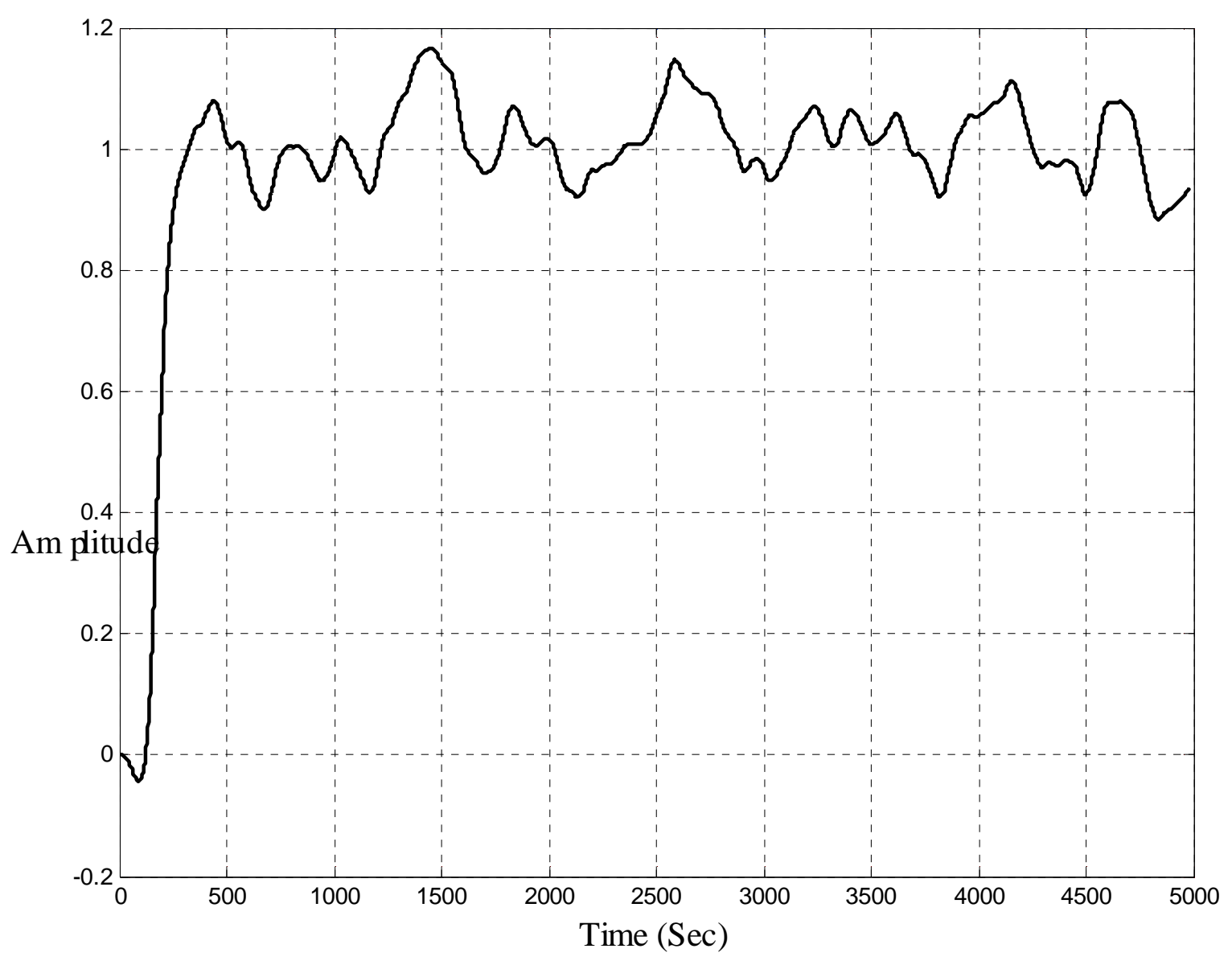

\section{Figure (7): Time response of the closed loop system using $R C G A$ with (-0.001) existence of amplitude disturbance}

\section{Conclusions:}

This paper presents the effect of RCGA to design a low order robust controller for an antenna tracking system. The robustness of this controller compare well with other controllers designed, recently, using $\mathrm{H} \infty$ loop shaping and the method of inequalities.

The numerical examples have been provided to demonstrate the merits and capabilities of the RGGA. It is noted that, the RCGA offered the same Gain margin as $\mathrm{H} \infty$ but it offered better settling time, rise time, peak time, over shoot percentage and Gain and Phase margin for each problem. The merits of RCGA over the $\mathrm{H} \infty$ loop shaping method is that RCGA produced 2nd order controller and, on that other hand, the $\mathrm{H} \infty$ produced 4th order controller. Farther more, the RCGA is very sample and easy to use for any numerical optimization problem. 


\section{References:}

[1] M. R. Said, "Robust optimal coordination approach with functional in-equality constraints", PHD thesis, Faculty of Engineering Alexandria university; September 1993.

[2] Adham Ahmed Abbas El- Mahallway. "Critical control system design ",MSC thesis, ,Faculty of Engineering Alexandria university, August 2001.

[3] E. M. Mekheal. "Robust Controller design using Hœ loop shaping \& the method of in-equlities ",MSC thesis, college of Engineering \& Technology ; October 2006.

[4] E, Mekheal,M.I.El-Singaby ,A. Khalil " Robust Controller Design using Ho Loop Shaping and the Method of Inequalities ",IEEE ISIE,Montreal,Quebec,Canada, July 2006.

[5] A. A. M. Madkour, M. A. Hossain, and K. P. Dahal "Intelligent Learning Algorithms for Active Vibration Control”, IEEE Trans. Contr. Syst. Technol., pp: 111-120, 2007.

[6] G. Himmelfarb, "Darwin and the Darwinian Revolution", Doubleaday and Company Inc., New York, 1959.

[7] D. B. Fogel, "Evolutionary computation", toward a new philosophy of machine intelligence: IEEE Press Piscataway, NJ, USA, 1995.

[8] M. D. Canada, "Genetics: What is Autosomal Recessive," http://www.muscle.ca/content/index.php?id=315, 2002.

[9] E. Richard and M. D. Robert, "Nelson Essentials of Pediatrics", 3 ed: W.B. Saunders Company, 1998

[10] A. A. M. Madkour, M. A. Hossain, and K. P. Dahal, "Modified Crossover Operator Approach for Evolutionary Optimization," presented at The Colloquium on Optimization for Control, University of Sheffield, UK, 2006 